\title{
BOUNDEDNESS FOR A CLASS OF FRACTIONAL CARLESON TYPE MAXIMAL OPERATOR
}

\section{XiAO Yu, HUIHUI Zhang AND XiAOMEI Wu}

Abstract. In this paper, the authors study the fractional Carleson type maximal operators $\mathscr{T}_{\beta}^{*}$ which is defined by

$$
\mathscr{T}_{\beta}^{*} f(x)=\sup _{\lambda}\left|\int_{\mathbb{R}^{n}} e^{i P_{\lambda}(y)} \frac{\Omega(y)}{|y|^{n-\beta}} f(x-y) d y\right|,
$$

where $0<\beta<n$ and $\Omega$ satisfies the $L^{q}$-Dini conditions with $1<q<\infty$. The authors prove the $L^{p} \rightarrow L^{p}$ boundedness of $\mathscr{T}_{\beta}^{*}$ under certain conditions.

Mathematics subject classification (2010): 42B20, 42B25.

Keywords and phrases: Fractional Carleson type maximal operator, Dini-condition, homogeneous kernel.

\section{REFERENCES}

[1] Calderón A. P., Weiss M. And Zygmund A., On the existence of singular integrals, Proc. Symp. Pure Math., AMS . 10 (1967), 56-73.

[2] CARLESON L., On convergence and growth of partial sums of Fourier series, Acta Math., 111(1960), 361-370.

[3] Colzano L., Hardy spcace on spheres, Ph. D. Thesis, Washington University, St. Louis, 1982.

[4] Ding Y. AND LiU H.H., $L^{p}$ boundedness of Carleson type maximal operators with nonsmooth kernels, Tohoku Math. J.(2), 63 (2012), 255-267.

[5] Ding Y. AND LiU H.H., Weighted $L^{p}$ boundedness of Carleson type maximal operators, Proc. Amer. Math. Soc., 140(8), (2012), 2739-2751.

[6] Ding Y. AND LU S.Z., Weighted norm inequalities for fractional integral operarors with rough kernel, Canad. J. Math. 50 (1998), 29-39.

[7] DuOAndikoetXeA J., Weighted norm inequalities for homogeneous singular integrals, Trans. Amer. Math. Soc., 336(1993), 869-880.

[8] Garcia-Cuerva, J. And Rubio de Francia, J.L., Weighted Norm Inequalities and Related Topics, Amsterdam: North-Holland, (1985).

[9] Hunt R.A., On the convergence of Fourier series, Orthogonal Expansions and Their Continuous Analogues, (Proc. Cont. Edwardsville, Ill., 1967), 235-255, Southern Illinois Univ. Press, Carbondale Ill., 1968.

[10] B. Muckenhoupt, Weighted norm inequalities for the Hardy maximal function, Trans. Amer. Math. Soc., 165(1972), 207-226.

[11] Muckenhoupt B. AND Wheeden R.L., Weighted norm inequalities for fractional integrals, Trans. Amer. Math. Soc. 192(1974), 261-274.

[12] P. SJÖLIN, Convergence almost everywhere of certain singular integral and multiple Fourier series, Ark. Mat. 9 (1971), 65-90.

[13] Stein E.M. And WAinger S., Oscillatory integrals related to Carleson's theorem, Math. Res. Lett. 8 (2001), 789-800. 